\title{
A Study of the Natural History of Vitreomacular Traction Syndrome by Optical Coherence Tomography.
}

Marie-Hélène Errera*, MD, Sidath E. Liyanage*‡, FRCOphth, Petros Petrou, MD, Pearse A. Keane, FRCOphth, Rene Moya, MD, Eric Ezra, FRCOphth, David G. Charteris, FRCOphth, Louisa Wickham ${ }^{\ddagger}$, FRCOphth.

Affiliation for all authors: Moorfields Eye Hospital NHS Trust, London, United Kingdom.

* joint first authors

${ }^{\ddagger}$ joint corresponding authors

Financial support: None

Conflict of interest statement: No conflicting relationship exists for any author

Running head: Natural history of VMTS using OCT

Address for reprints: Miss Louisa Wickham, Vitreoretinal department, Moorfields Eye Hospital NHS Trust, City Road, London, United Kingdom. EC1V 2PD. 


\section{Abstract}

Purpose: To examine the natural history of Vitreomacular Traction Syndrome (VMTS) in the absence of other ocular comorbidities.

Design: Retrospective clinical case series.

Participants: 183 eyes of 159 patients diagnosed with VMTS with no other ocular comorbidity.

Methods: Patients with VMTS were identified from an Optical Coherence Tomography (OCT) database at Moorfields Eye Hospital, London. Sequential OCT scans and patient notes were reviewed over a minimum period of 6 months. Data collected included patient demographics, best corrected visual acuity and OCT features of vitreo-macular adhesion. Contingency tests and binary logistic modeling were used to identify baseline predictors of stability and progression.

Main outcome measures: The rates of spontaneous resolution (defined by release of traction), progression to full-thickness macular hole or surgical intervention were analysed.

Results: Presenting visual acuity was $0.3 \pm 0.3 \log M A R$ units. The mean length of follow-up was $17.4 \pm 12.1$ months. During this period, VMTS persisted in $60 \%$ and 
resolved in $20 \%$ (occurring on average at 15 months). Of the remainder, $12 \%$ developed a macular hole and $8 \%$ elected to proceed with surgery for symptoms. Focal adhesion of $<1500 \mu \mathrm{m}$ was present in $87 \%$. A premacular membrane with macular pucker (PMM) was present in $20 \%$. With persistent VMTS, vision and central foveal thickness remained unchanged. The relative risk of resolution increased in those cases with better presenting visual acuities, lesser foveal thicknesses and no associated PMMs; in those cases with resolution there was a significant improvement in vision.

Conclusion: VMTS persists in the majority of patients but despite this, visual acuities did not deteriorate significantly over the study period unless patients developed a full-thickness macular hole or required surgical intervention for symptoms. Resolution spontaneously occurred in $20 \%$, with an improvement in vision.

\section{Introduction}

Vitreomacular traction syndrome (VMTS) forms part of a spectrum of diseases with abnormal vitreomacular adhesion. This condition was initially thought to be relatively uncommon, as only gross cases were identified by clinical examination. The development of optical coherence tomography (OCT) led to an increase in its diagnosis and revived interest in VMTS.

VMTS is characterized by anomalous posterior vitreous detachment (PVD) accompanied by anatomic distortion of the fovea. This may include the presence of 
pseudocysts, macular schisis, cystoid macular edema and subretinal fluid. Clinically, symptoms can vary from none to distortion and/or reduced visual acuity. In some cases visual acuity may progressively deteriorate resulting in visions of $20 / 200$ (logMAR 1.0) or less. ${ }^{1}$ This may also be accompanied by macular hole formation.

More recently, the International Vitreomacular Traction Study group has developed a classification system that aids in the characterization of this abnormal interaction between the vitreous and macula. ${ }^{2}$ In so doing, this has allowed more consistent reporting of vitreomacular interface disorders.

There is a paucity of natural history data relating to VMTS within the published literature. Controversy exists regarding the exact rate of spontaneous resolution in eyes with VMTS. Some reports suggest that the rate of spontaneous PVD in this condition is uncommon, with Hikichi et al. reporting a PVD rate of $11 \%$ over a fiveyear period; other authors have observed a higher rate of approximately $50 \%$ in a follow-up of around 9 months..$^{1,3,4}$ To further confound this issue of resolution, Reibaldi et al. highlight a case with a ten year history of non-resolving severe VMTS with no resultant anatomical or functional damage. ${ }^{5}$

The limited natural history data is surprising given that therapeutic interventions are offered to patients with VMTS, including vitrectomy and more recently, enzymatic vitreolysis. ${ }^{6}$ In cases where spontaneous resolution of VMTS fails to occur, vitrectomy may be offered to patients who complain of symptoms of reduced vision and/or distortion. ${ }^{7}$ Knowledge of the rate of spontaneous improvement is important 
for patients making the decision regarding surgery, as well as providing doctors with sufficient information for informed consent. Following the advent of intravitreal injections of gas or pharmacologic agents such as ocriplasmin to induce vitreolysis, cases of mild yet symptomatic VMTS are now considered for intervention..$^{6,8}$ This further supports the need for more robust natural history data.

This study describes the natural history of VMTS over a minimum period of 6 months. To our knowledge, this large series differs from others as it concerns VMTS in the absence of other ocular comorbidities and utilizes an independent accredited OCT grader to assess OCT features of VMTS, with a follow up of more than 6 months.

\section{Methods}

This is a retrospective cohort study which was approved by the local Ethics Review Board committee and adhered to the tenets set forth in the Declaration of Helsinki. 183 eyes in 159 patients with a diagnosis of vitreomacular traction syndrome (VMTS) were identified from a database containing all patients imaged with OCT between 2009 and 2011 at Moorfields Eye Hospital, London. The inclusion criterion was the presence of vitreomacular traction on $\mathrm{OCT}$, as defined by the International Vitreomacular Traction Study Group: evidence of perifoveal vitreous cortex detachment from the retinal surface; macular attachment of the vitreous cortex within a 3-mm radius of the fovea; and association of the attachment with distortion of the foveal surface, intraretinal structural changes, elevation of the fovea above 
the RPE, or a combination thereof, but no full-thickness interruption of all retinal layers. ${ }^{2}$ Cases with lamellar macular holes were excluded from this study. All patients had a minimum follow up period of six months, unless there was either resolution, progression to a full-thickness macular hole or surgery for symptoms. Exclusion criteria included coexisting retinal disease; previous history of retinal detachment; ocular inflammation; and proliferative retinal disease.

The International Vitreomacular Traction Study Group grading system classifies VMTS into focal $(<1500 \mu \mathrm{m})$ or broad $(\geq 1500 \mu \mathrm{m})$ groups dependent on maximal horizontal surface adhesion. ${ }^{2}$ To complement this, we utilized a system described by John et al. to catalogue the intraretinal OCT findings on presentation (Figure 1). Here, grade 1 is defined as incomplete cortical vitreous separation with foveal attachment. The additional presence of intraretinal cysts/clefts denotes grade 2 and in grade 3 a foveal photoreceptor detachment is also present. ${ }^{9}$

The following data were collected on each patient at the time of inclusion: patient demographics, best corrected visual acuity and OCT features of VMTS, including macular thickness, maximal horizontal surface adhesion and intraretinal changes. Imaging was performed with either Topcon 3D-OCT (Topcon, Japan) or Spectralis OCT (Heidelberg Engineering, Germany). For the Topcon 3D-OCT, a macular volume scan was performed for each eye consisting of 128 horizontal B-scans, centered through the fovea. For the Spectralis OCT, a similar horizontal raster scan was performed, centered on the fovea and covering a $6 \times 6 \mathrm{~mm}^{2}$ area. Patients underwent a series of OCT scans spanning a period of more than 6 months. All OCT scans were 
graded, then validated by a single, reading center-certified, OCT grader masked to all clinical information at the time of grading.

The rate of spontaneous posterior vitreous detachment (PVD), as defined by resolution of traction and improvement of anatomic distortion of the fovea, was documented. Over the study period some patients elected to have surgery. Surgery in this unit was offered for progression to macular hole or for symptoms of significant distortion and/or reduction of vision present with both eyes open. The Snellen visual acuity was documented at the final follow up visit.

\section{Data analysis and statistic methods}

Data were analyzed with descriptive and frequency statistics. Snellen visual acuities were converted to logMAR units for analysis. The application of parametric and nonparametric tests was dependent on normality, as determined by using the D'Agostino and Pearson test. The statistical distribution of groups was compared using either the unpaired t test (two-tailed), Mann-Whitney (two-tailed) and KruskalWallis test (for comparison of more than two groups). The Wilcoxon test was used to compare paired groups while the Chi-squared test was used to compare categorical variables. The relative risk was used to calculate the strength of association between categorical variables, as the prevalence of VMTS is less than $10 \% \cdot{ }^{10} \mathrm{~A} P$ value less than 0.05 was considered statistically significant. In graphs, the error bars depict standard deviation. Binary logistic regression models for different outcomes were fit 
with potential baseline predictors: age, visual acuity, gender, grade, adhesion, PMM and foveal thickness. The former two variables are continuous data while the remainder are nominal data. Models were refined by using a stepwise backward elimination of non-significant $(P \geq 0.05)$ variables. A $P$ value greater than 0.05 was used to confirm the validity of the model using the 'goodness of fit' tests. Statistical analysis was performed using Minitab 16 (Minitab Inc., USA) and Prism 6.0 (Graphpad Software Inc., USA).

\section{Results}

\section{Initial examination}

A diagnosis of vitreomacular traction syndrome (VMTS) was identified in 183 eyes of 159 patients (Table 1), with a preponderance (70\%) of women among cases. The mean age at the time of presentation was $72 \pm 10$ years (mean \pm standard deviation; range, 45 - 95 years). Both eyes were affected in 28 patients (18\%). Of the remainder, 77 (48\%) presented with VMTS in the right eye and $82(52 \%)$ in the left eye. The mean baseline visual acuity was $0.3 \pm 0.3 \log$ MAR units, with $18 \%$ of cases having a visual acuity of $0.0 \log$ MAR units or better at presentation. There was no statistically significant difference in baseline visual acuity when the focal and broad groups were compared (Mann-Whitney test) or between grades 1, 2 or 3 (KruskalWallis test) (Figure 2). 


\section{OCT Features at presentation}

Patients were classified as having focal adhesion if the extent of vitreomacular adhesion was equal to or less than $1500 \mu \mathrm{m}$. Focal adhesion at the macula was present in 159 (87\%) of the 183 eyes, with a mean width of $542 \pm 270 \mu \mathrm{m}$ (63 - 1407 $\mu \mathrm{m})$. In the remaining 24 eyes with broad adhesion, the average width of adhesion was $2188 \pm 375 \mu \mathrm{m}(1623-2526 \mu \mathrm{m})$.

The mean foveal thickness at presentation was $317 \pm 96 \mu \mathrm{m}$ in the focal group, statistically significantly less than the initial foveal thickness of $410 \pm 113$ in the broad group ( $P<0.001$; Mann-Whitney test). When the intraretinal OCT findings were considered, similar measurements revealed average baseline foveal thicknesses of $313 \pm 82,333 \pm 108$ and $289 \pm 100$ for grades 1, 2 and 3 respectively, with a statistically significant difference between grades 2 and 3 only $(P<0.05$; Kruskal-Wallis test with Dunn's multiple comparisons post hoc analysis) (Figure 3).

An premacular membrane with associated macular pucker (PMM) was present in 37 eyes $(20 \%)$, with a statistically significant association with degree of adhesion $\left(\chi^{2}=\right.$ $\left.44_{\mathrm{df}=1}, P<0.0001\right)$ : cases with a broad adhesion have 5.6 relative risk $(95 \% \mathrm{Cl} 3.5-$ 9.1) of having a PMM when compared to those cases with a focal adhesion (Figure 4).

\section{Outcomes}


The average length of follow-up was $17.4 \pm 12.1$ months (range, 6 - 48 months). Of the 183 eyes included, there was no resolution in the majority (60\%). Resolution of VMTS occurred in 36 patients (20\%) with a mean time to resolution of $15 \pm 14$ months (range, 1 - 48 months); the incidence of resolution was 0.14/person-year. Over the study period, 23 patients (12\%; incidence, 0.09/person-year) developed a full-thickness macular hole and 15 patients (8\%; incidence, 0.06/person-year) elected to proceed with surgery for worsening symptoms. On average, this progression occurred at $19 \pm 15$ months and $13 \pm 8$ months respectively. There was no significant differences between the times to resolution, progression or surgery (Kruskal-Wallis test) (Figure 5).

The overall initial visual acuity of $0.3 \pm 0.3 \log$ MAR units was unchanged at the end of the study, with no statistically significant change in individual cases (Wilcoxon test). In cases with resolution of VMTS, the mean visual acuity was $0.1 \pm 0.2 \log M A R$ units, representing a significant improvement of $0.1 \pm 0.2 \operatorname{logMAR}$ units $(P<0.01$; paired $\mathrm{t}$ test). In patients with stable VMTS at the end of the follow up period, there was no significant change in the visual acuity with an average visual acuity of $0.3 \pm 0.2$ logMAR units (Wilcoxon test). Development of a full-thickness macular hole resulted in a significant decrease in vision to $0.7 \pm 0.2 \log$ MAR units $(P<0.001$; paired t test $)$. Conversely, surgery for symptoms of VMTS significantly improved vision from $0.8 \pm$ $0.8 \log M A R$ units to $0.4 \pm 0.2 \log$ MAR units $(P<0.01$; Wilcoxon test) (Figure 6). 


\section{Presenting visual acuity}

While the baseline visual acuity is independent of whether a case with VMTS remains stable or changes $\left(\chi^{2}=2 \mathrm{df}=1\right)$, there is a significant association between this baseline vision and either resolution or progression to a full-thickness macular hole or surgery, if there is change $\left(\chi^{2}=6 \mathrm{df}=1, P<0.05\right)$ (Figure 7A). Here, those presenting with vision better than or equal to $0.3 \log M A R$ units have 2 relative risk ( $95 \% \mathrm{Cl} 1.1$ 3.6) of resolution and 0.6 relative risk ( $95 \% \mathrm{Cl} 0.4-0.9)$ of progression than those with worse vision. In both groups, there was a significant improvement in vision with resolution ( $\leq 0.3$ : $P<0.05$; Wilcoxon test; $>0.3: P<0.01$; paired t test). With progression, a significant decrease in vision was only noted in the group with presenting vision $\leq 0.3 \operatorname{logMAR}$ units $(P<0.01 ;$ paired t test). Asymptomatic VMTS (visual acuity better than or equal to 0.0 logMAR units) was not a significant factor in determining whether a case either remained stable or resolved or progressed $\left(\chi^{2}=\right.$ $2.9 \mathrm{df}=1$ ) (Figure 7B).

\section{Presenting foveal thickness}

Similar to presenting visual acuity, the foveal thickness at presentation did not influence the stability of the condition $\left(\chi^{2}=1 \mathrm{df}=1\right)$. If there was either resolution or progression to a full-thickness macular hole or surgery, the presenting foveal thickness was a significant factor $\left(\chi^{2}=8 \mathrm{df}=1, P<0.01\right)$ (Figure 7 C). Eyes with increased foveal thickening of less than $400 \mu \mathrm{m}$ have 3.1 relative risk $(95 \% \mathrm{Cl} 1.1$ - 
8.9) of resolution and 0.5 relative risk $(95 \% \mathrm{Cl} 0.3-0.8)$ of progression than those with thicker foveas. Despite this association, there was no significant difference between presenting and final visual acuities in both groups (Wilcoxon test).

Mean foveal thickness at the end of follow up was $316 \pm 105 \mu \mathrm{m}$. In patients with no resolution of VMTS over the study period, the average final foveal thickness was 334 $\pm 91 \mu \mathrm{m}$, indicating no significant change in the foveal thickness when compared to presentation (Wilcoxon test). Resolution of VMTS produced a significant improvement in foveal thickness with a reduced mean final foveal thickness of $242 \pm$ $79 \mu \mathrm{m}(P<0.05$; Wilcoxon test).

\section{Vitreoretinal adhesion and retinal features}

There was no significant association between the type of horizontal adhesion (focal vs. broad) and whether a case of VMTS either persisted or resolved or progressed (evolution to full-thickness macular hole or surgery for symptoms) $\left(\chi^{2}=1 \mathrm{df}=2\right)$ (Figure 7D). Likewise, the presence of intraretinal cysts/clefts or foveal photoreceptor detachment (grades 1, 2 or 3) did not significantly influence persistence, resolution or progression in VMTS $\left(\chi^{2}=4.5 \mathrm{df}=4\right)$ (Figure 7E). However, in this case series, a focal adhesion was present in all cases of VMTS that progressed to a full-thickness macular hole. 


\section{Presence of premacular membrane with associated macular pucker}

Classically, VMTS exerts an anteroposterior or axial traction on the macula, whereas macular pucker is associated with tangential macular traction. Therefore, analysis was undertaken to determine whether the presence of a PMM with macular pucker (and the differing vector of traction) affected outcome in such cases, as compared to those with VMTS only. Although the presence of a PMM with macular pucker is independent of whether a case remains stable or changes $\left(\chi^{2}=2.4 \mathrm{df}=1\right)$, its absence becomes a significant factor if there is change $\left(\chi^{2}=7.1 \mathrm{df}=1, P<0.01\right)$ (Figure 8). Those cases with isolated VMTS have 2.3 relative risk ( $95 \% \mathrm{Cl} 1.1-5.0)$ of resolution and 0.5 relative risk $(95 \% \mathrm{Cl} 0.3-0.8)$ of progression than those cases with a PMM with macular pucker.

\section{Independent predictors of differing outcomes in isolated VMTS}

When the outcome of resolution is compared to those of stability, development of a full-thickness macular hole or requiring surgery for symptoms, the only significant baseline variable is age (Table 2). However, the effect of this variable was minimal, with increasing age reducing the odds of resolution by a factor of 0.94 .

Age at presentation was also a predictor of stability, increasing the odds of stable VMTS by a factor of 1.07. In this model, baseline visual acuity had a greater impact of 
whether a case remained stable. Poorer vision decreased the odds of a case remaining stable by a factor of 0.14 .

Similarly, presenting visual acuities were important significant variables when modeling the outcome of either development of full-thickness macular hole or the requirement for surgery. Poor vision increased the odds of these outcomes by factors of 12.89 and 15.52 respectively. However, the presence of a PMM with pucker at baseline had contrasting effects on whether a case developed a macular hole or required surgery for symptoms, reducing the odds by a factor of 0.08 in the former and increasing the odds by a factor of 15.63 in the latter.

\section{Discussion}

Here, we present natural history data of both symptomatic and asymptomatic patients diagnosed with vitreomacular traction syndrome (VMTS), as defined by the International Vitreomacular Traction Study (IVMTS) group. ${ }^{2}$ In this study, the mean age of patients presenting with this condition was 72 years, with women comprising the majority (70\%). On average, the baseline visual acuity was $0.3 \pm 0.3 \log M A R$ units. A PMM was present in $20 \%$ of cases. These characteristics are consistent with those noted in a slightly larger retrospective study (203 eyes with VMTS) by Stalmans. ${ }^{11}$ 
However, there are notable differences between our study and that published by Stalmans. Firstly, our paper analyses isolated VMTS in cases with no ocular comorbidity, while approximately $13 \%$ of cases with VMTS had diabetes in the latter study. Secondly, the degree of adhesion, foveal thickness and intraretinal changes were not analysed by Stalmans; in our study, these factors were independently assessed by a reading centre-certified OCT grader. This enabled us to statistically analyse the impact of the factors on differing outcomes for VMTS.

The similarity in baseline characteristics may have influenced the consistency in outcomes between the above studies. For example, the ReCoVit study cites an outcome visual acuity of $0.3 \pm 0.4$ LogMAR units in the VMTS only group, with $23 \%$ experiencing spontaneous resolution, $26 \%$ needing surgery for worsening symptoms and $5 \%$ developing full-thickness macular holes. ${ }^{11}$ This compares favorably with our outcome data which shows a final visual acuity of $0.3 \pm 0.3$ and respective rates of $20 \%, 12 \%$ and $8 \%$. The agreement of these two large independent studies, together with resolution rates of $20-30 \%$ in other studies, ${ }^{9,12}$ suggests the possibility of a defined pattern for the outcomes of isolated VMTS.

This study also emphasizes the timing of resolution or progression of VMTS. On average, these events occurred after 12 months in both cases. The mean time of resolution is identical to that seen in a large pan-American retrospective case series. ${ }^{12}$ Dimopoulos et al. analysed a subset of VMTS with focal adhesion and no PMM and found that $55 \%$ of cases resolved after more than 12 months, and highlighted this in relation to the final 6 month endpoint for resolution used in the 
enzymatic vitreolysis trials. ${ }^{6,13}$ Together, this data supports a more conservative temporal estimate of resolution when counseling patients.

Resolution of VMTS in our cohort of cases caused a statistically significant improvement in vision, in concordance with other studies. ${ }^{11,12}$ Similarly, surgery for symptoms of VMTS resulted in an improvement in vision, while development of a full-thickness macular hole decreased vision. Others have used logistic regression modeling to identify cases which resolve or progress to vitrectomy. ${ }^{11,14}$ We performed contingency tests to identify baseline characteristics associated with stability, resolution or progression in VMTS, with the latter being defined as the development of a full-thickness macular hole or surgical intervention for symptoms.

Interestingly, these tests showed that cases presenting with vision better than or equal to 0.0 LogMAR units did not have higher than expected rates of spontaneous resolution. Further analysis revealed that this 'threshold' was 0.3 LogMAR units, with those with better vision having higher rates of resolution and lower rates of progression than those cases with poorer vision. When this concept was applied to foveal thickness at baseline, another 'threshold' of $400 \mu \mathrm{m}$ was discovered. Again, cases with thinner foveas have a higher relative risk of resolution and a lower relative risk of progression.

Other studies have not compared the natural history of focal and broad VMTS, as defined by the IVMTS group. ${ }^{2}$ Randomized controlled trials investigating the therapeutic effect of enzymatic vitreolysis on VMTS resolution have shown a 
differential in the rate of resolution between the focal (37\%) and broad (14\%) groups. ${ }^{6}$ Our statistical analysis did not reveal an association between this variable and either rate of resolution or progression. In an attempt to classify the retinal architectural changes secondary to VMTS, John et al. devised three grades dependent on the presence of retinal cysts/clefts and foveal photoreceptor detachments on OCT. These authors, and Wu et al. subsequently, showed that there was no statistical difference in outcomes between the grades. ${ }^{9,12}$ We graded our dataset using John et al.'s system and again failed to discern any differing outcomes, suggesting that the retinal architectural changes in VMTS are independent of outcome.

Our data also shows that the presence of a PMM is significantly associated with broad VMTS. This observation was noted by Odrobina et al., who postulated that the extent of the vitreous attachment could influence spontaneous regression of vitreofoveal traction. ${ }^{3}$ While not conclusive, results from the previously mentioned randomized controlled trials suggests that the presence of a PMM affects outcomes: among patients without a PMM, VMTS resolution occurred in $37 \%$ of the enzymatic vitreolysis group and in $14 \%$ of the placebo group. In patients with a PMM, these rates reduced to $9 \%$ and $2 \%$ respectively. ${ }^{6}$ These findings are supported by contingency tests on our outcomes, which show that cases without a PMM and macular pucker have a higher relative risk of resolution and a lower relative risk of progression. 
Vitreoschisis is an abnormal split in the posterior vitreous cortex; a PVD in this setting results in the outermost layer of split vitreous cortex remaining attached to the retina. It can be identified using OCT combined with scanning laser ophthalmoscopy by the 'lambda' or ' $Y$ ' sign and was present in $53 \%$ and $42 \%$ of eyes with macular holes and pucker respectively. ${ }^{15}$ Using similar criteria, vitreoschisis occurred in $6 \%$ of our cases. Of the 11 cases of VMTS with vitreoschisis seen, only 1 case resolved and 4 required surgery for symptomatic pucker.

In our analyses, while age was a significant predictor of whether a case of isolated VMTS resolved, remained stable or progressed to a full-thickness macular hole, it had minimal effect on these outcomes. In contrast, visual acuity at baseline had a profound effect on whether a case remained stable or required surgery for symptoms. This reflects clinical practice, where patients with poorer vision are more likely to require surgery for symptoms. However, it is notable that a worse presenting visual acuity increases the odds of a case developing a macular hole. The presence of a PMM was associated with a greater odds of requiring surgery for symptoms. This predictor also decreases the odds of developing a full-thickness macular hole.

There are limitations to our study, which include its retrospective nature; additional prospective data on long-term outcomes is still required. Visual acuity is used as measure of visual function, and does not reflect metamorphopsia due to retinal distortion. The latter is often a major symptom in VMTS and can be objectively measured using either three-dimensional threshold Amsler grid or M-charts. ${ }^{16}$ This 
study did not evaluate natural history of VMTS with regards to this symptom. It

should be noted that the number of patients with asymptomatic VMTS that present to clinics appears low. This raises the possibility of creating a reporting bias, where this potentially 'milder' part of the VMTS spectrum is under-represented, skewing outcomes towards the more severe symptomatic cases.

\section{References}

1. Hikichi T, Yoshida A, Trempe CL. Course of vitreomacular traction syndrome. Am J Ophthalmol 1995;119:55-61.

2. Duker J, Kaiser PK, Binder S, et al. The International Vitreomacular Traction Study Group Classification of Vitreomacular Adhesion, Traction, and Macular Hole. Ophthalmology 2013;120:2611-2619.

3. Odrobina D, Michalewska Z, Michalewski J, et al. Long-term evaluation of vitreomacular traction disorder in spectral-domain optical coherence tomography. Retina (Philadelphia, Pa) 2011;31:324-331.

4. Charalampidou S, Nolan J, Beatty S. The natural history of tractional cystoid macular edema. Retina (Philadelphia, Pa) 2012;32:2045-2051.

5. Reibaldi M, Avitabile T, Uva MG, et al. Ten Years of Severe Vitreomacular Traction Syndrome without Functional Damage Demonstrated by Optical Coherence Tomography. Case Rep Ophthalmol Med 2011;2011:931038.

6. Stalmans P, Benz MS, Gandorfer A, et al. Enzymatic vitreolysis with ocriplasmin for vitreomacular traction and macular holes. N Engl J Med 2012;367:606-615.

7. Koerner F, Garweg J. Vitrectomy for macular pucker and vitreomacular traction syndrome. Doc Ophthalmol 1999;97:449-458.

8. Rodrigues IA, Stangos AN, McHugh DA, Jackson TL. Intravitreal injection of expansile perfluoropropane $(\mathrm{c}(3) \mathrm{f}(8))$ for the treatment of vitreomacular traction. Am J Ophthalmol 2013;155:270-276.e2.

9. John VJ, Flynn HW, Smiddy WE, et al. Clinical course of vitreomacular adhesion managed by initial observation. Retina (Philadelphia, Pa) 2014;34:442-446.

10. Meuer SM, Myers CE, Klein BEK, et al. The epidemiology of vitreoretinal interface 
abnormalities as detected by spectral-domain optical coherence tomography: the beaver dam eye study. Ophthalmology 2015;122:787-795.

11. Stalmans P. A retrospective cohort study in patients with tractional diseases of the vitreomacular interface (ReCoVit). Graefes Arch Clin Exp Ophthalmol 2016;254:617-628.

12. Wu L, Zas M, Berrocal MH, et al. Anatomical and functional outcomes of symptomatic idiopathic vitreomacular traction: A Natural History Study From the Pan American Collaborative Retina Study Group. Retina (Philadelphia, Pa) 2016;36:19131918.

13. Dimopoulos S, Bartz-Schmidt K-U, Gelisken F, et al. Rate and timing of spontaneous resolution in a vitreomacular traction group: should the role of watchful waiting be re-evaluated as an alternative to ocriplasmin therapy? $\mathrm{Br} \mathrm{J}$ Ophthalmol 2015;99:350-353.

14. Almeida DRP, Chin EK, Rahim K, et al. Factors associated with spontaneous release of vitreomacular traction. Retina (Philadelphia, Pa) 2015;35:492-497.

15. Gupta P, Yee KMP, Garcia P, et al. Vitreoschisis in macular diseases. Br J Ophthalmol 2011;95:376-380.

16. Nguyen JH, Yee KM, Sadun AA, Sebag J. Quantifying Visual Dysfunction and the Response to Surgery in Macular Pucker. Ophthalmology 2016;123:1500-1510.

\section{Figure legends}

Figure 1. Retinal OCT scans depicting John et al.'s grading of retinal architecture in VMTS: 1 (A) vs. 2 (B) vs. 3 (C). (D) shows an example of a broad adhesion subtype of the International Vitreomacular Traction Study group subtypes.

Figure 2. Graphs showing presenting visual acuities for International Vitreomacular Traction Study group subtypes (focal vs. broad adhesions) and John et al.'s grading of retinal architecture (1 vs. 2 vs. 3). 
Figure 3. Graphs showing initial foveal thickness for International Vitreomacular Traction Study group subtypes (focal vs. broad adhesions) and John et al.'s grading of retinal architecture (1 vs. 2 vs. 3 ) $(* * * P<0.001$ using Mann-Whitney test; $* P<0.05$ using Kruskal-Wallis test).

Figure 4. Graph showing number of cases with either focal or broad adhesion presenting with a premacular membrane with associated macular pucker (PMM).

Figure 5. Time taken for cases to resolve, develop full-thickness macular holes (FTMH) or require surgery.

Figure 6. Graph showing the initial and final visual acuities for groups in which VMTS remained stable, resolved and progressed to either full-thickness macular hole (FTMH) or surgery (** $P<0.01$ and $* * * P<0.001$; Resolved and FTMH using paired $\mathrm{t}$ test; Surgery using Wilcoxon test).

Figure 7. Graphs showing number of cases which either persist / change or resolve / progress to full-thickness macular holes or surgery, in relation to various baseline factors. 